\title{
Ações e práticas de enfermagem frente ao paciente psiquiátrico atendido em um hospital de urgência e emergência de Porto Velho-RO
}
Actions and pratices of nursing in respect of the psychiatric patient carried out in na urgent emergency and emergency hospital of Porto Velho - RO

Acciones y prácticas de enfermería frente al paciente psiquiátrico atendido em um hospital de urgencia y emergencia de Porto Velho - RO

Francisca Juscileide do Nascimento Azevedo Pimenta ${ }^{1 \star}$, Marcela Milrea Araújo Barros.

\section{RESUMO}

Objetivo: Compreender as práticas de enfermagem frente ao paciente psiquiátrico atendido na emergência clínica de um pronto socorro no município de Porto Velho-RO. Métodos: Trata-se de uma pesquisa descritiva de abordagem qualitativa. A amostra foi constituída por 16 enfermeiros e técnicos de enfermagem, atuantes na emergência clínica. Resultados: Os resultados evidenciaram que os profissionais apresentam fragilidades, não somente em compreender, mas também em lidar com as emergências psiquiátricas. Referem à presença do medo, vulnerabilidade e insegurança frente ao paciente psiquiátrico. Fragilidades na qualificação dos profissionais, diante das crises psiquiátricas, pode ser um elemento propulsor para ações violentas e sem fins terapêuticos, o que dificulta o vínculo com o paciente e assistência a ser prestada. Conclusão: É preciso que as condições no ambiente de trabalho favoreçam a qualidade do serviço prestado, aliado a qualificações, através de educação permanente, favorecendo a qualidade no atendimento aos pacientes em crises.

Palavras-Chave: Enfermagem, Paciente, Psiquiatria, Emergência.

\begin{abstract}
Objective: To understand the nursing practices in front of the psychiatric patient attended in the emergency room of an emergency room in the city of Porto Velho-RO. METHODS: This is a qualitative descriptive research. The sample consisted of 16 nurses and nursing technicians working in the clinical emergency. Results: The results showed that the professionals present weaknesses, not only in understanding, but also in dealing with psychiatric emergencies. They refer to the presence of fear, vulnerability and insecurity in front of the psychiatric patient. Fragility in the qualification of professionals, in the face of psychiatric crises, can be a propelling element for violent and non-therapeutic actions, which makes it difficult to bond with the patient and assistance to be provided. Conclusion: It is necessary that the conditions in the work environment favor the quality of the service provided, together with qualifications, through permanent education, favoring quality in the care of patients in crises.

professionals understand about the subject psychiatric crises, favoring the quality in the care of patients in crisis.
\end{abstract}

Keywords: Nursing, Patient, Psychiatry, Emergency.

1União das Escolas Superiores de Rondônia (UNIRON), Porto Velho - RO. *E-mail: juscileideazevedo@uol.com.br

SUBMETIDO EM: 6/2019

ACEITO EM: 7/2019

PUBLICADO EM: 7/2019 


\section{RESUMEN}

Objetivo: Comprender las prácticas de enfermería frente al paciente psiquiátrico atendido en la emergencia clínica de un pronto socorro en el municipio de Porto Velho-RO. Métodos: Se trata de una investigación descriptiva de abordaje cualitativo. La muestra fue constituida por 16 enfermeros y técnicos de enfermería, actuantes en la emergencia clínica. Resultados: Los resultados evidenciaron que los profesionales presentan fragilidades, no sólo en comprender, sino también en lidiar con las emergencias psiquiátricas. Se refiere a la presencia del miedo, vulnerabilidad e inseguridad frente al paciente psiquiátrico. Las fragilidades en la cualificación de los profesionales, ante las crisis psiquiátricas, puede ser un elemento propulsor para acciones violentas y sin fines terapéuticos, lo que dificulta el vínculo con el paciente y la asistencia a ser prestada. Conclusión: Es necesario que las condiciones en el ambiente de trabajo favorezcan la calidad del servicio prestado, aliado a calificaciones, a través de educación permanente, favoreciendo la calidad en las atenciones a los pacientes en crisis.

Palabras clave: Enfermería, Paciente, Psiquiatría, Emergencia.

\section{INTRODUÇÃO}

Emergências psiquiátricas podem ser definidas como alterações agudas do pensamento, do humor, do comportamento ou das relações sociais que requerem uma intervenção imediata, devido à possibilidade de evolução rápida para um resultado deletério. Entre os possíveis resultados negativos incluem-se sofrimento psíquico significativo, perda da autonomia, comprometimento do papel social e risco potencial ou evidente à integridade psíquica e física do indivíduo ou de outras pessoas (DEU-BEM CM et al., 2017).

Segundo a Organização Mundial da Saúde (OMS) 2012, os transtornos mentais acometem mais de 450 milhões de pessoas e respondem por $12,3 \%$ das causas de enfermidades e invalidez. Mesmo que as autoridades acordem para o problema, o número deve chegar a 562 milhões em 2020. Isso ocasionará uma demanda reprimida impressionante nos serviços de psiquiatria, fato já estabelecido no Brasil de hoje.

O campo e emergência psiquiátrica é conflituoso, sendo indispensável à intervenção imediata de uma equipe multiprofissional que esteja treinada adequadamente, no intuito de evitar maiores prejuízos à saúde do indivíduo, ou de eliminar possíveis riscos à sua vida ou à de terceiros (DEU-BEM CM et al., 2017).

A Enfermagem em saúde mental no decorrer de sua história acumulou conhecimentos empíricos e, na maioria das vezes, seus profissionais têm realizado as ações baseadas em normas e rotinas repetidas e também atuado sem uma reflexão da sua prática, mesmo com as modificações do perfil da clientela, do avanço tecnológico e da organização dos próprios profissionais de enfermagem (CANABRAVA DS et al., 2012).

A emergência de um hospital geral recebe diariamente casos distintos, desde o sujeito que sentiu uma precordialgia à vítima de colisão, aquele que sofreu queda da própria altura ou que ingeriu drogas em excesso. Mais não deve ser esquecido que dentre todos exemplos opostos podemos ter associado o transtorno psíquico (PAES MR e MAFTUM MA, 2013).

Nem sempre os serviços de emergência dispõem de uma estrutura para o recebimento e as condutas necessária para o paciente com algum tipo de transtorno. Não obstante, a sociedade, os familiares e muitos profissionais de saúde veem a crise, na maioria dos casos, como uma demanda urgente quando começa a afetar a rotina social (BONFADA D e GUIMARÃES J, 2012).

De acordo com o Ministério da Saúde (MS) BRASIL (2006), o Sistema Único de Saúde (SUS) é um sistema público e universal baseado nos princípios da equidade, integralidade da atenção e controle social. Ele é executado nos Estados, municípios e em cada unidade de saúde através de uma atenção de qualidade e humanizada. Partindo da premissa da equidade frente ao paciente psiquiátrico. Isso não significa que todos mereçam o mesmo tratamento, pois para se ter equidade deve-se conhecer as diferenças e reconhecer a necessidade de tratar desigualmente os desiguais. 
Os profissionais de enfermagem são considerados como uma categoria que possui o contato mais intenso com os pacientes, esses trabalhadores representam a maior categoria que compõem a instituição hospitalar. Nesta perspectiva, a equipe de enfermagem precisa exercer suas condutas de forma segura e eficaz, pois o processo da reforma psiquiátrica exige cada vez mais a qualificação técnica e teórica dos trabalhadores do setor da saúde, necessitando, portanto, de uma equipe coesa, e que possua capacitação para tal (HOLANDA GS, et al.,2016).

O interesse pela pesquisa se deu pela experiência vivida durante a prática hospitalar de enfermagem na emergência clínica. De maneira empírica, foi observada a dificuldade da equipe de enfermagem em realizar atendimento ao paciente psiquiátrico. Considerando o paciente como parte central desse processo, é de fundamental importância discutir os aspectos que se relacionem a necessidade de um grupo preparado, responsável e organizado.

A questão norteadora desta pesquisa é: Como a equipe de enfermagem atua frente ao paciente psiquiátrico na emergência clínica? Essa pesquisa tem como objetivo geral compreender as ações e práticas de enfermagem frente ao paciente psiquiátrico atendido na emergência clínica de um pronto socorro em município de Porto Velho-RO.

\section{MÉTODOS}

Trata-se de uma pesquisa de abordagem qualitativa e descritiva a partir do uso de questionário com questões abertas realizada com a equipe de enfermagem do setor da emergência clínica do Hospital e Pronto Socorro João Paulo em Porto Velho, Rondônia.

A pesquisa foi composta pela totalidade de profissionais dos turnos manhã, tarde e noite, sendo: enfermeiros e técnicos de enfermagem que atuavam na emergência clínica. A amostra final compreendeu 16 profissionais que aceitaram participar voluntariamente do estudo, considerados os critérios de inclusão e exclusão.

Garantimos o anonimato dos participantes cujos nomes ficaram no sigilo, através do uso de códigos definidos por letras e números (Enfermeiros EN1, EN2 e Técnicos de Enfermagem TE1, TE2, por exemplo). A pesquisa foi submetida e aprovada pelo Comitê de Ética e Pesquisa (CEP) da União Educacional do Norte Ltda - UNINORTE com número de CAAE: 91105118.7 .0000 .8028 e Número do Parecer: 2.814.507. Foram respeitadas as disposições contidas na Resolução de № 466/2012 do Conselho Nacional de Saúde (CNS) que trata das pesquisas com seres humanos.

\section{RESULTADOS E DISCUSSÕES}

$\mathrm{Na}$ análise empírica dos dados, tendo como base relatos semelhantes e convergentes, a partir da matriz de síntese, foram elencados dois núcleos temáticos: A equipe de enfermagem diante da crise psiquiátrica e a educação permanente e sua importância para a qualificação da equipe de enfermagem.

\section{A equipe de enfermagem diante da crise psiquiátrica}

A crise é caracterizada como uma situação de emergência em que as pessoas apresentam dificuldades em resolver determinados problemas habituais. Podemos dizer que uma pessoa está em sofrimento mental, ou seja, em crise, quando há um desequilíbrio emocional no qual as pessoas não conseguem lidar com tal situação, sejam elas de perdas ou ameaças de algo significativo (FERNANDES MA, et al., 2016).

As limitações frente às crises podem contribuir para que a equipe de enfermagem desenvolva sentimentos relacionados ao medo, vulnerabilidade e insegurança, conforme se observa na fala dos participantes da pesquisa:

"Sinto-me com medo se ser agredido no momento de um surto." (TE 1)

"Vulnerável, desprotegido e receoso no momento de uma crise." (TE 2) 
Para Paes MR e Maftum MA (2013), mesmo com a quebra de paradigmas, nos últimos anos, em saúde mental, os profissionais ainda mostram medo excessivo em relação ao paciente em sofrimento psíquico. Este fato pode estar relacionado à subjetividade inerente à saúde mental, termo de difícil definição, podendo os pacientes expressar diversas emoções e comportamentos, os quais geralmente são classificados como aberrantes, desviantes, incapacitantes e de risco para si e para os outros, onde para Nobre AMD (2012) a sensação de medo, sentida pelos profissionais de saúde, prejudica no julgamento clínico, podendo conduzir a uma assistência com quantidades desnecessárias de medicamentos sedativos e de contenções físicas e mecânicas.

A contenção química consiste na utilização de fármacos para impedir comportamentos destrutivos. A via oral é preferível para a aplicação da contenção química, sempre que possível e enquanto o paciente estiver colaborativo. Em casos de não cooperação, o medicamento poderá ser administrado por via intramuscular. (MANTOVANI C, et al., 2010). Ou seja, há possibilidade do paciente com sintomas agudos, manifestar comportamento agressivo e agitação psicomotora, de modo que as abordagens de acolhimento pela comunicação verbal não sejam suficientes.

Para Fernandes MA, et al. (2016), a contenção física poderá ser um procedimento válido no atendimento às emergências psiquiátricas, enquanto que, Araujo EM, et al. (2010) nos traz a utilização da contenção mecânica, quando a física não é suficiente, tendo por objetivo restringir os movimentos do paciente agressivo e que está agitado, limitando seus movimentos quando se oferece perigo para si e para os profissionais de saúde, onde se é utilizado faixas para um relaxamento progressivo, ocorrendo uma diminuição da agressividade e agitação corporal do paciente.

De acordo com os profissionais, a dificuldade no atendimento, no momento em que o paciente está em crise, a melhor alternativa acaba sendo a utilização de contenções química e mecânica para minimizar os danos que podem acontecer no momento, evidenciados nas falas abaixo:

\section{"Contenção química e mecânica é a única alternativa de minimizar os danos no momento da crise." (TE 4)}

Conforme Resolução do Conselho Federal de Enfermagem n.ำ 427/2012 Art. 1ํㅡ, os profissionais da enfermagem, excetuando-se as situações de urgência e emergência, somente poderão empregar a contenção mecânica do paciente sobsupervisão direta do enfermeiro e, preferencialmente, em conformidade com protocolos estabelecidos pelas instituições de saúde, públicas ou privadas, a que estejam vinculados.

A partir da perspectiva dos profissionais, identificou-se que a maioria possui limitações no conhecimento para resolutividade dos problemas relacionados a crises psiquiátricas, pois se apresentam inseguros para prestar a assistência de enfermagem, corroborando com o que afirmam Willich JQ, et al. (2014) no qual a sensação de insegurança nos profissionais, pode levar a contenções mecânicas e medicamentosas desnecessárias por não conhecerem a forma adequada de manejo durante o cuidado. Resultados convergem com estudo realizado por Holanda GS, et al. (2015), em Ribeirão Preto-SP, que teve como objetivo conhecer 0 atendimento realizado pela equipe de enfermagem ao paciente psiquiátrico em um pronto atendimento e, um dos resultados, foi a insegurança, durante as crises, relatada pelos profissionais.

Outro estudo realizado por Bonfada D e Guimarães J (2015), na cidade de Natal-RN, objetivou discutir o atendimento prestado aos indivíduos em crises psiquiátricas pelos profissionais de saúde do Serviço de Atendimento Móvel de Urgência (SAMU), descreveram que as condutas realizadas estavam associadas à prática manicomial, pois os profissionais faziam o uso da força coercitiva, sendo esta exercida até com o auxílio de policiais militares. A contenção física realizada sem a indicação correta podem trazer danos físicos, como necrose, trombos, fraturas, aspiração e asfixia e psíquicos para os pacientes (PAES MR, MAFTUM MA, 2013), podendo ser empregada como último recurso nos episódios de agressividade, uma vez que devem ser priorizadas medidas menos restritivas e, em nenhum caso, a contenção física deve ocorrer como punição ou intimidação (BARK J, et al., 2012). Nesse contexto, os cuidados frente às emergências psiquiátricas são realizados de forma fragmentada, o que pode influenciar na tomada de decisão de maneira errônea frente ao paciente em crise psiquiátrica. 
Segundo os profissionais (EM 5 e TE 5), ao realizar o atendimento ao paciente psiquiátrico, um pequeno grupo da equipe atuante na emergência clínica tem investido na tentativa de humanizar a assistência, conforme as falas abaixo:

"Independentemente da situação, ele é um ser humano, tento atendê-lo de forma mais humanizada possível com as condições oferecidas." (EN 5)

A Política Nacional de Humanização (BRASIL, 2006), constituída de forma transversal às demais políticas de saúde, contribui para a qualidade da atenção e gestão do SUS. Sua criação se deve à necessidade de avanço e qualificação do sistema nacional de saúde, na relação e nos processos de atenção ao usuário, bem como no trabalho de gestores e trabalhadores da área, reconhecendo a singularidade e a capacidade criadora de cada sujeito envolvido.

Não há uma única concepção e forma de se realizar o acolhimento. O acolhimento, conforme propõe a $\mathrm{PNH}$, deve ser entendida, ao mesmo tempo, como diretriz ética, estética, política, constitutivas dos modos de se produzir saúde e como ferramenta tecnológica relacional de intervenção na escuta, na construção de vínculo, na garantia do acesso com responsabilização e na resolutividade dos serviços (GUEDES MVC, 2013).

A sobrecarga da equipe de enfermagem constitui-se um dos fatores que influenciam na qualidade de vida no trabalho que, por sua vez, apresenta relação com os níveis de insatisfação associados ao serviço. Para Boagaert J, et al. (2013), a sobrecarga no trabalho está relacionada diretamente ao desenvolvimento de exaustão emocional e influencia indiretamente na qualidade do cuidado, onde os níveis de sobrecarga interferem na qualidade dos serviços prestados aos pacientes psiquiátricos. Na fala do profissional (EN 2) a superlotação e a falta de estrutura, são os fatores que mais interferem para a realização de uma boa assistência.

"Sobrecarregado devido à superlotação do hospital e a falta de estrutura mínima para além do cuidado a preservação da privacidade do usuário."

Em hospitais psiquiátricos, especialmente, a equipe de enfermagem vivencia de forma constante um clima de tensão emocional e de frustração, uma vez que prestam assistência a pacientes crônicos e portadores de transtornos mentais graves que apresentam poucas mudanças e com condições de trabalho muitas vezes precárias que geram insatisfação no trabalho, sobrecarga física e psíquica (CANABRAVA DS, et al., 2012).

\section{A educação permanente e sua importância para a qualificação da equipe de enfermagem}

A Política Nacional de Educação Permanente em Saúde (PNEPS) foi instituída pelo Ministério da Saúde por meio da Portaria 198, de fevereiro de 2004 (BRASIL, 2004), no sentido de viabilizar as necessidades de formação e de desenvolvimento dos trabalhadores da saúde, além de aprimorar constantemente a qualidade do cuidado à saúde. Mas, foi a portaria do Ministério da Saúde n. 1.996, publicada em 2007 (BRASIL, 2007), que redefiniu as diretrizes e estratégias para a implementação da PNEPS, adequando-as às diretrizes operacionais do Pacto pela Saúde.

A deficiência na qualificação dos profissionais no desempenho de suas funções em situações de crise, pode se tornar um elemento propulsor de ações violentas e sem fins terapêuticos, impossibilitando o estabelecimento de vínculo e assim dificultando a assistência (BONFADA D e GUIMARÃES J, 2012).

O Hospital e Pronto Socorro, como cenário de estudo, recebe diariamente diversos tipos de pacientes, inclusive, pacientes em surtos psicóticos.

Os profissionais relataram que não se sentem preparados em prestar assistência diante das emergências psiquiátricas:

"Não fui e nem me sinto preparada para tal assistência, que é essencial para o cuidado de qualidade." (TE 4)

"Não me sinto preparada, inclusive já fui até agredida." (EN 3) 
A não abordagem do assunto crises psiquiátricas é vista como uma das deficiências na formação dos enfermeiros e técnicos em enfermagem e que repercute em sua vida profissional, levando a uma dificuldade em prestar assistência aos pacientes que estão em crises psicóticas.

Corroboram com esta pesquisa um estudo feito por Nobre AMD (2012) em um hospital universitário de Campina Grande. Verificou-se, na maioria dos participantes, o auto relato de despreparo para tal cuidado, prejudicando a assistência de enfermagem. Entende-se que a educação permanente contribui no processo de consolidação da reforma psiquiátrica para que os profissionais tenham maior conhecimento sobre as formas de cuidado, planejamento e intervenções em pacientes com crise. As estratégias de EPS devem ser baseadas e estruturadas a partir da problematização do processo de trabalho e ser compreendida como processo contínuo, no qual o foco é a reinserção social deste indivíduo e adesão ao tratamento (KONDO EH et al., 2011).

Nesta perspectiva, ações de educação permanente tem alta significância no ambiente do atendimento hospitalar, um espaço de grandes possibilidades de saberes, pois considera a aprendizagem significativa, ou seja, que os indivíduos, a partir dos problemas que se evidenciam no ambiente do trabalho, podem refletir e discutir em equipe as necessidades educacionais para aprimorar o processo de trabalho (VEDANA KGG, 2016). Dos 16 participantes da pesquisa, 14 responderam que precisam de qualificações, pois se sentem inabilitados para atenderem esse público que, a cada dia, cresce no âmbito hospitalar:

"Necessitamos de mais capacitações no atendimento ao paciente psiquiátrico, já que somos hospital que recebemos todos os tipos de casos." (EN 4)

Para Duxbury J e Wright K (2011) a equipe deve ter, a sua disposição, protocolos e rotinas que facilitem uma abordagem coesa e organizadora. Além disso, discussões, a respeito da temática "crises psiquiátricas", devem ser feitas com periodicidade com o objetivo de melhorar o atendimento a estes pacientes. Tal processo sugere que as práticas dos profissionais estejam pautadas na reflexão crítica e nas práticas reais e em ação na rede de serviços. A educação permanente tem, como lógica, a produção de novos pactos e acordos coletivos, para fortalecer as ações das equipes, provocando transformações e mudanças institucionais, além de práticas técnicas profissionais e sociais (SANTOS TO et al., 2017).

O hospital de estudo possui um Núcleo de Educação Permanente (NEP) atuante, no entanto, nas ações de educação em saúde, encontram grande dificuldade na adesão dos profissionais às capacitações ofertadas, corroborando com o que diz o autor Sardinha PLL et al., (2013), onde em um estudo feito no Rio Grande do Sul, apontaram dificuldades quanto à realização de ações de EPS nas instituições de saúde, principalmente, no que consta à baixa adesão dos profissionais nas atividades educativas, ocasionado pela resistência deles, por se considerarem detentores dos conhecimentos necessários a promoção de saúde.

Em estudo realizado por Miccas FL e Batista SHSE (2014), é referido o alto número de profissionais que não participam das atividades de EPS por estarem em seu horário de trabalho, dificultando, assim, a sua implementação em instituições hospitalares. Mendonça FF e Nunes EFPA (2011), em estudo no Hospital do Paraná, traz a estratégia de inclusão das atividades de EPS no cotidiano dos profissionais como parte das atividades laborais. Esses encontros foram sistemáticos e previstos em escala, com programação elaborada conforme as necessidades provenientes das unidades de atendimento do hospital.

\section{CONCLUSÃO}

O estudo evidenciou dificuldades enfrentadas pelos profissionais diante das situações de crise, envolvendo sentimentos, como medo, tristeza, impotência e insegurança. Estes sentimentos são comuns, pois a empatia e relacionamento terapêutico envolvem o processo de cuidado, possibilitando que o profissional possa imaginar-se na situação de usuário. Torna-se necessário que estes sentimentos sejam trabalhados de forma construtiva, possibilitando um cuidado focado na dignidade, respeito, afeto e acolhida das necessidades dos usuários. Torna-se necessário que as condições no ambiente de trabalho favoreçam a qualidade do serviço prestado que, aliado a qualificações, através da educação permanente, os profissionais se sensibilizem, contribuindo para a qualidade do atendimento ao usuário. 


\section{REFERÊNCIAS}

1. ARAUJO EM, et al. Inquérito sobre o uso de contenção física em um hospital psiquiátrico de grande porte no Rio de Janeiro J. bras. psiquiatr. vol.59 no.2 Rio de Janeiro 2010.

2. BARK J, et al. Contenção mecânica - quais intervenções evitam episódios de contenção mecânica - uma revisão sistemática. - A Systematic Review. Perspectives in Psychiatric Care v. 48, n.2, p.83-94, 2012.

3. BOAGAERT J, et al. Uma comparação controlada randomizada de reclusão e contenção mecânica em ambientes hospitalares Psychiatric Services, Washington, v. 62, n. 11, p. 1310-1317, 2013.

4. BONFADA D; GUIMARÃES J. Serviço de atendimento móvel de urgência e as urgências psiquiátricas. Psicol. estud. vol.17 no.2 Maringá Apr. /June 2012.

5. BRASIL. Portaria nำ 198 GM/MS, de 13 de fevereiro de 2004. Institui a Política Nacional de Educação Permanente em Saúde como estratégia do Sistema Único de Saúde para a formação e o desenvolvimento de trabalhadores para o setor e dá outras providências. Diário Oficial da União 2004.

6. BRASIL. Portaria no 1.996 GM/MS, de 20 de agosto de 2007. Dispõe sobre as diretrizes para a implementação da Política Nacional de Educação Permanente em Saúde. Diário Oficial da União 2007.

7. BRASIL, Ministério da Saúde. Secretaria de Atenção à Saúde. Núcleo Técnico da Política Nacional de Humanização. HumanizaSUS: documento base para gestores e trabalhadores do SUS. 3.ed. Brasília: Ministério da Saúde, 2006.

8. CANABRAVA DS, et al. Diagnostico e intervenções à pessoa com transtorno mental com base na consulta de enfermagem. Cogitare Enfermagem. 2012 Out/Dez; 17(4):661-8.

9. DEU-BEM CM, et al. Emergências psiquiátricas: manejo de agitação psicomotora e avaliação de risco suicida, (Ribeirão Preto, Online.) 2017;50(Supl.1), jan-fev.:98-112.

10. DUXBURY, J.; WRIGHT, K. As enfermeiras devem restringir pacientes violentos e agressivos London, v. 107, n. 9, p. 22-25, Mars 2011.

11. FERNANDES, MA et al. Cuidados de enfermagem ao paciente psiquiátrico na urgência de um hospital geral. Rev Enferm UFPI. 2016 Abr-Jun;5(2):41-45.

12. GUEDES MVC. Acolhimento em um serviço de emergência: percepção dos usuários. Rev. Bras. Enferm, Brasília, vol. 66, n.1, p. 66(1):31-37, 2013.

13. HOLANDA GS, et al. Atuação do Enfermeiro em emergência psiquiátrica. 2 congressos brasileiro em saúde 2016 .

14. KONDO EH, et al. Abordagem da equipe de enfermagem ao usuário na emergência em saúde mental em um pronto atendimento. Rev. esc. enferm. USP 2011, vol.45, n.2, pp.501-507.

15. MANTOVANI C, et al. Manejo de paciente agitado ou agressivo. Revista Brasileira de Psiquiatria, São Paulo, v. 32 , S. 2, p. 96-103, 2010.

16. MENDONÇA FF; NUNES EFPA. Necessidades e dificuldades de tutores e facilitadores para implementar a política de educação permanente em saúde em um município de grande porte no estado do Paraná, Brasil. Interface: Comun Saúde Educ.2011;15(38):871-82.

17. MICCAS FL; BATISTA SHSE. Educação permanente em saúde: metassíntese, Rev Saúde Pública 2014;48(1):170185.

18. NOBRE AMD. Assistência de enfermagem em sofrimento psíquico Monografia (Especialização em Saúde Mental e Atenção Psicossocial) - Universidade Estadual da Paraíba, Centro de Ciências Biológicas e Saúde, 2012.

19. ORGANIZAÇÃO MUNDIAL DA SAÚDE (OMS). Prevenção do suicídio: Um manual para profissionais da saúde em atenção primária. Transtornos mentais e comportamentais. Departamento de Saúde Mental. Genebra, 2000.

20. PAES MR; MAFTUM MA. Percepção da equipe de enfermagem de um pronto atendimento sobre a pessoa com transtorno mental. Rev Enferm UFSM 2013 Set/Dez;3(3):461-469.

21. SANTOS TO et al. A Importância da Educação Continuada para Profissionais de Saúde que atuam no Atendimento Hospitalar International Nursing Congress maio 2017.

22. SARDINHA PLL et al. Educação permanente, continuada e em serviço: desvendando seus conceitos Enfermería Global № 29 Enero 2013 Página 32.

23. VEDANA KGG. Urgencia e emergência psiquiátrica. EERP-USP 2016.

24. WILLRICH JQ, et al. Da violência ao vínculo: construindo novos sentidos para a atenção à crise. Revista Brasileira de Enfermagem. vol.67, n.1, p. 97-103, 2014. 